УДК 517.957

Яновская О.С., Северо-Каваказский федеральный университет, Сурнева О.Б. г. Ставрополь, Россия olenka_yan@mail.ru

\title{
НЕЛИНЕЙНОЕ УРАВНЕНИЕ, ОБЛАДАЮЩЕЕ ОПЕРАТОРОМ РАССЕЯНИЯ ТРЕТЬЕГО ПОРЯДКА
}

Введение:

большинство дифференциальных уравнений, связанных с солитонной математикой, получены с помощью операторного уравнения Лакса или уравнения нулевой кривизны, которые являются условием совместности пары линейных дифференциальных систем. Глубоко и всесторонне изучен случай, когда для получения таких уравнений использовались системы второго порядка. Повышение порядка систем ведет к сильно переопределенным условиям. В работе изучается возможность использовать линейные системы третьего порядка.

Материалы и методы исследований:

использованы методы построения уравнений в частных производных с применением операторного уравнения Лакса с дисференциальными операторами первого порядка и матричными коэффрициентами $3 \times 3$.

Результаты исследования:

Обсуждение и заключения:

Ключевые слова:

определены необходимые и достаточные условия, накладываемые на параметры и функции, входящие в матрицы-коэфффициенты, при которых коммутатор двух дифференциальных операторов представляет оператор умножения. Показано, что уравнение Лакса сводится к системе девяти уравнений, порядок которой можно понизить и свести к одному нелинейному уравнению в частных производных.

авторами продемонстрированы два примера вывода нелинейных уравнений и определение их пары Лакса. В первом примере главный дифференциальный коэфффициент рассматривается в виде нижнетреугольной матрицы, а во втором случае постоянная матрица имеет диагональный вид. В результате получены уравнения второго порядка с логарифмической нелинейностью.

нелинейные уравнения в частных производных, операторное уравнение Лакса, пара Лакса, условие совместности системы дифференциальных уравнений. 
Yanovskaya O.S., North-Caucasus Federal University, Stavropol, Russia

Surneva O.B. olenka_yan@mail.ru

\section{NONLINEAR EQUATION WITH THE THIRD ORDER SCATTERING OPERATOR}

Introduction:

Materials and

methods of research: methods for constructing partial differential equations using the Lax operator equation with differential operators of the first order and matrix coefficients of $3 \times 3$ were used.

Research results:

Discussion and conclusions:

Keywords:

most of the differential equations associated with soliton mathematics are obtained using the Lax operator equation or the zero-curvature equation, which are the compatibility condition for a pair of linear differential systems. The case in which second-order systems were used to obtain such equations was studied in depth and comprehensively. Increasing the order of systems leads to highly overdetermined conditions. The possibility of using third-order linear systems is being studied.

necessary and sufficient conditions imposed on the parameters and functions included in the matrix coefficients, under which the commutator of two differential operators represents the multiplication operator. It is shown that the Lax equation reduces to a system of nine equations, the order of which can be reduced and reduced to one nonlinear partial differential equation.

the authors demonstrated two examples of the derivation of nonlinear equations and the determination of their Lax pair. In the first example, the main differential coefficient is considered as a lower triangular matrix, and in the second case the constant matrix has a diagonal form. As a result, secondorder equations with a logarithmic nonlinearity are obtained.

nonlinear partial differential equations, Lax operator equation, Lax pair, compatibility condition for a system of differential equations.

\section{Введение}

Большинство известных нелинейных уравнений, обладающих парой Лакса $[1,7]$, являются либо точно интегрируемые или уравнения, допускающие богатые классы точных решений. Поэтому наибольший интерес с практической точки зрения вызывают те исследования, которые способствуют развитию новых математических методов анализа нелинейных систем уравнений и в частности теорию, связанную с идеями солитонной математики.

Малоисследованным, но очень обширным классом уравнений являются многокомпонентные уравнения. Для этих уравнений полных списков пока не найдено. Вместе с тем, уравнения этого типа важны с прикладной точки зрения. Покажем, как предложенная схема модифицируется на случай многокомпонентных нелинейных уравнений, в частности, уравнений 3-х волнового взаимодействия $[2,3,4]$. Постановка задачи в этом случае может быть сформулирована следующим образом. 
Операторное уравнение Лакса имеет вид

$$
L_{t}=[L, A]=L A-A L,
$$

где операторы могут быть различной природы (дифференциальные, интегральные, матрицы, проектирующие и др.). Идея, лежащая в основе работы Лакса [6], заключается в том, что нелинейное уравнение (1) является условием совместности системы линейных уравнений:

$$
\begin{aligned}
& L \varphi=\mu \varphi, \\
& \varphi_{t}=-A \varphi,
\end{aligned}
$$

для оператора $L$ поставлена спектральная задача ( $\varphi$ - собственная функция, $\mu$ - собственное значение оператора $L$ ), оператор $A$ определяет эволюцию собственных функций по времени $t$.

Рассмотрим частный случай [5], когда $L, A$ дифференциальные операторы первого порядка, заданные в пространстве вектор-функций и имеющие вид

$$
L=\alpha \frac{\partial}{\partial x}+U, \quad A=\beta \frac{\partial}{\partial x}+V
$$

где $\quad \alpha=\left(\alpha_{i j}\right), \beta=\left(\beta_{i j}\right), i, j=1,2,3-$ постоянные матрицы $3 \times 3, U=$ $\left(v_{i j}(x, t)\right), \mathrm{i}, \mathrm{j}=1,2,3$ - матрицы $3 \times 3$ с компонентами, зависящими от $x$ и $t$.

Лемма 1. Правая часть уравнения Лакса (1) представляет собой дифференщиальный оператор второго порядка вида:

$[L, A]=[\alpha, \beta] \frac{\partial^{2}}{\partial x^{2}}+([U, \beta]+[V, \alpha]) \frac{\partial}{\partial x}+[U, V]+\alpha V_{x}-\beta U_{x}$.

Следствие. $\quad$ Если матрицы $\alpha, \beta, V, U$ удовлетворяют следующим условиям

$$
[\alpha, \beta]=0, \quad[U, \beta]+[\alpha, V]=0,
$$

то коммутатор представляет оператор умножения

$$
[L, A]=[U, V]+\alpha V_{x}-\beta U_{x} .
$$

Лемма 2. $\quad$ Условия (5) выполняются тогда и только тогда, когда параметры $\alpha_{i j}, \beta_{i j}, i, j=1,2,3$, удовлетворяют равенствам

$$
\alpha_{i j} \beta_{j i}+\alpha_{i k} \beta_{k i}-\beta_{i j} \alpha_{j i}-\beta_{i k} \alpha_{k i}=0, i, j, k=1,2,3, i \neq j \neq k
$$




$$
\sum_{j=1}^{3}\left[\alpha_{i j} \beta_{j k}-\beta_{i j} \alpha_{j k}\right]=0, \quad i, k=1,2,3, i \neq k,
$$

а функциональные компоненты $u_{i j}(x, t), v_{i j}(x, t), i, k=1,2,3$, удовлетворяют равенствам

$$
\begin{aligned}
& u_{i j} \beta_{j i}+u_{i k} \beta_{k i}-\beta_{i j} u_{j i}-\beta_{i k} u_{k i}+\alpha_{i j} v_{j i}+\alpha_{i k} v_{k i}-v_{i j} \alpha_{j i}-v_{i k} \alpha_{k i}=0, \\
& i, j, k=1,2,3, \quad i \neq j \neq k \\
& \sum_{j=1}^{3}\left[u_{i j} \beta_{j k}-\beta_{i j} u_{j k}+\alpha_{i j} v_{j k}-v_{i j} \alpha_{j k}\right]=0, \quad i, k=1,2,3, i \neq k .
\end{aligned}
$$

\section{Материалы и методы исследований}

Лемма 3: $\quad$ Если параметры матрии $\alpha=\left(\alpha_{i j}\right), \beta=\left(\beta_{i j}\right), i, j=1,2,3$ и функциональные матрицы $U=\left(u_{i j}(x, t)\right), V=\left(v_{i j}(x, t)\right), i j=1,2,3$ такие, что

$\alpha_{12}=\alpha_{13}=\alpha_{23}=0, \quad-\alpha_{11}=\alpha_{22}=\alpha_{33}$,

$\beta_{12}=\beta_{13}=\beta_{23}=0, \quad \beta_{11}=3 k \alpha_{11}, \beta_{22}=\beta_{33}=k \alpha_{11}$,

$\beta_{21}=k \alpha_{21}, \beta_{31}=k \alpha_{31}, \beta_{32}=k \alpha_{32}$,

остальные $\alpha_{11}, \alpha_{21}, \alpha_{31}, \alpha_{32}, k$ - произвольные параметры отличные от нуля, а функиии $u_{i j}(x, t) \neq 0, i, j=1,2,3$ удовлетворяют связям:

$$
\begin{gathered}
u_{33}=u_{22}+\frac{2}{k} v_{11}, \\
v_{11}=-v_{22}=v_{33}, \quad v_{1 j}=k u_{1 k}, \quad v_{j 1}=k u_{j 1}+\frac{\alpha_{k 1}}{2 \alpha_{11}}\left(2 v_{11}+k\left(u_{22}-u_{11}\right)\right), j=2,3, \\
v_{23}=k u_{23}, \quad v_{32}=k u_{32}+\frac{\alpha_{32}}{2 \alpha_{11}}\left(2 v_{11}+k\left(u_{22}-u_{11}\right)\right),
\end{gathered}
$$

тогда выполняются условия (7) - (10) .

Теорема 1: Операторное уравнение Лакса с дифференциальными операторами первого порядка (4) с коэффиииентами, удовлетворяющими леммам 1, 2, 3, эквивалентно нелинейному уравнению с частными производными 


$$
\begin{aligned}
& \frac{\alpha_{21} \alpha_{32}}{2 \alpha_{11} k}\left(u_{13}-\ln u_{13}\right)_{z z}=\left(\frac{\alpha_{31}^{2}}{\alpha_{11}}-\frac{\alpha_{32}^{2} \alpha_{21}^{2}}{4 \alpha_{11}^{3}}\right) u_{13 z}+\frac{\alpha_{32}^{2} \alpha_{21}^{2}}{4 \alpha_{11}^{3}} u_{13 z} \ln u_{13}+\frac{\alpha_{31}}{k} \frac{u_{13 z z}}{u_{13}}-\alpha_{32} \alpha_{21} \frac{u_{13 x z}}{u_{13}}, \\
& \text { где } \quad 2 k \alpha_{11} \frac{\partial}{\partial x}+\frac{\partial}{\partial t}=\frac{\partial}{\partial z} .
\end{aligned}
$$

Доказательство: Коэффициенты операторов (4) удовлетворяют леммам $1,2,3$, для нахождения результата уравнения Лакса (1) воспользуемся равенством

$$
\begin{aligned}
& u_{i j t}=u_{i 1} v_{1 j}+u_{i 2} v_{2 j}+u_{i 3} v_{3 j}-v_{i 1} u_{1 j}-v_{i 2} u_{2 j}-v_{i 3} u_{3 j}+\alpha_{i 1} v_{1 j x}+\alpha_{i 2} v_{2 j x}+\alpha_{i 3} v_{3 j x}- \\
& -\beta_{i 1} u_{1 j x}-\beta_{i 2} u_{2 j x}-\beta_{i 3} u_{3 j x}, \quad i, j=1,2,3 .
\end{aligned}
$$

Легко заметить, что можно выделить группы производных и для более компактной записи в уравнениях ввести следующую замену переменных (16).

Уравнение Лакса (1) даст систему:

$$
\begin{gathered}
u_{11 z}=\left(\frac{\alpha_{21}}{2 \alpha_{11}} u_{12}+\frac{\alpha_{31}}{2 \alpha_{11}} u_{13}\right)\left(2 v_{11}+k\left(u_{22}-u_{11}\right)\right)+\alpha_{11} v_{11 x}-k \alpha_{11} u_{11 x}, \\
u_{12 z}=\left(u_{13} \frac{\alpha_{32}}{2 \alpha_{11}}-u_{12}\right)\left(2 v_{11}+k\left(u_{22}-u_{11}\right)\right), \\
\quad-u_{13 z}=\left(2 v_{11}+k\left(u_{22}-u_{11}\right)\right) u_{13}, \\
u_{21 z}=\left[u_{21}+\left(u_{22}-u_{11}\right) \frac{\alpha_{21}}{2 \alpha_{11}}+\frac{\alpha_{31}}{2 \alpha_{11}} u_{23}\right]\left(2 v_{11}+k\left(u_{22}-u_{11}\right)\right)-\frac{\alpha_{21}}{2} k\left(u_{22}+u_{11}\right)_{x}, \\
u_{22 z}=\left(u_{23} \frac{\alpha_{32}}{2 \alpha_{11}}-\frac{\alpha_{21}}{2 \alpha_{11}} u_{12}\right)\left(2 v_{11}+k\left(u_{22}-u_{11}\right)\right)+\alpha_{11} v_{11 x}+k \alpha_{11} u_{22 x}, \\
u_{31 z}=\left(u_{32} \frac{\alpha_{21}}{2 \alpha_{11}}-u_{21} \frac{\alpha_{32}}{2 \alpha_{11}}+\frac{\alpha_{31}}{2 \alpha_{11}}\left[2 v_{11}+k\left(u_{22}-u_{11}\right)\right]+u_{31}\right)\left(2 v_{11}+k\left(u_{22}-u_{11}\right)\right)+ \\
+\frac{\alpha_{32} \alpha_{21}}{2 \alpha_{11}}\left(2 v_{11}+k\left(u_{22}-u_{11}\right)\right)_{x}-\frac{\alpha_{31}}{2} k\left(u_{22}+u_{11}\right)_{x}, \\
u_{32 z}=\left[v_{11} \frac{\alpha_{32}}{k \alpha_{11}}-\frac{\alpha_{31}}{2 \alpha_{11}} u_{12}\right]\left(2 v_{11}+k\left(u_{22}-u_{11}\right)\right)-2 \alpha_{32} v_{11 x}-\frac{\alpha_{32}}{2} k\left(3 u_{22}-u_{11}\right)_{x}, \\
u_{22 z}+\frac{2}{k} v_{11 z}-\alpha_{11} v_{11 x}=-\left(\frac{\alpha_{31}}{2 \alpha_{11}} u_{13}+u_{23} \frac{\alpha_{32}}{2 \alpha_{11}}\right)\left(2 v_{11}+k\left(u_{22}-u_{11}\right)\right)+\alpha_{11} k u_{22 x} .
\end{gathered}
$$

* Введена новая нумерация в системе из девяти уравнений. Первая цифра означает порядковый номер в системе, вторая цифра обозначает этап преобразований. В дальнейшем не все уравнения в системе могут участвовать в преобразованиях. 
Система (1-I) - (9-I) содержит связь девяти неизвестных функций, функцию $u_{13}(x, z)$ будем считать отличной от нуля вместе с ее производными и постараемся выразить остальные неизвестные функции через нее.

Разделим третье уравнение (3-I) системы на $u_{13}$, откуда находим

$$
v_{11}=\frac{k}{2}\left(u_{11}-u_{22}\right)-\frac{1}{2}\left(\ln u_{13}\right)_{z} .
$$

Подставим полученное выражение для функции $v_{11}$ в оставшиеся равенства системы. Шестое уравнение полученной системы легко интегрируется, и функция $u_{23}$ явно выражается через функцию $u_{13}$

$$
u_{23}=\frac{\alpha_{21}}{2 \alpha_{11}} u_{13}
$$

постоянная интегрирования, для простоты дальнейшего изложения, принята равной нулю (во всех дальнейших шагах этот факт больше не оговаривается).

Уравнение (2-I), с учетом (18), является линейным относительно $u_{12}$, при этом функция $u_{13}$ считается известной, после его интегрирования, находим

$$
u_{12}=-\frac{\alpha_{32}}{2 \alpha_{11}} u_{13} \ln u_{13}
$$

Подставим полученные значения в остальные уравнения системы:

$$
\begin{aligned}
& u_{11 z}=u_{13 z}\left(\frac{\alpha_{32} \alpha_{21}}{4 \alpha_{11}^{2}} \ln u_{13}-\frac{\alpha_{31}}{2 \alpha_{11}}\right)-\alpha_{11} \frac{k}{2}\left(u_{11}+u_{22}\right)_{x}-\frac{1}{2} \alpha_{11}\left(\ln u_{13}\right)_{z x}, \\
& u_{21 z}=-\left(\ln u_{13}\right)_{z}\left[u_{21}+\left(u_{22}-u_{11}\right) \frac{\alpha_{21}}{2 \alpha_{11}}+\frac{\alpha_{31} \alpha_{21}}{4 \alpha_{11}^{2}} u_{13}\right]-\frac{\alpha_{21}}{2} k\left(u_{22}+u_{11}\right)_{x}, \\
& u_{22 z}=\frac{k}{2} \alpha_{11}\left(u_{11}+u_{22}\right)_{x}-\frac{1}{2} \alpha_{11}\left(\ln u_{13}\right)_{z x}-\frac{\alpha_{32} \alpha_{21}}{4 \alpha_{11}^{2}} u_{13 z}\left(1+\ln u_{13}\right), \\
& u_{31 z}=-\left(\ln u_{13}\right)_{z}\left(u_{32} \frac{\alpha_{21}}{2 \alpha_{11}}-u_{21} \frac{\alpha_{32}}{2 \alpha_{11}}-\frac{\alpha_{31}}{2 \alpha_{11} k}\left(\ln u_{13}\right)_{z}+u_{31}\right)- \\
& -\frac{\alpha_{32} \alpha_{21}}{2 \alpha_{11}}\left(\ln u_{13}\right)_{z x}-\frac{\alpha_{31}}{2} k\left(u_{22}+u_{11}\right)_{x}, \\
& u_{32 z}=-\left(\ln u_{13}\right)_{z}\left[\frac{\alpha_{32}}{2 \alpha_{11}}\left(u_{11}-u_{22}\right)-\frac{\alpha_{32}}{2 k \alpha_{11}}\left(\ln u_{13}\right)_{z}\right]-\frac{\alpha_{31} \alpha_{32}}{4 \alpha_{11}^{2}} u_{13 z} \ln u_{13}+ \\
& +\alpha_{32}\left(\ln u_{13}\right)_{z x}-\frac{1}{2} \alpha_{32} k\left(u_{11}+u_{22}\right)_{x}, \\
& u_{11 z}-\frac{1}{k}\left(\ln u_{13}\right)_{z z}+\alpha_{11} \frac{1}{2}\left(\ln u_{13}\right)_{z x}=\left(\frac{\alpha_{31}}{2 \alpha_{11}}+\frac{\alpha_{21} \alpha_{32}}{4 \alpha_{11}^{2}}\right) u_{13 z}+\alpha_{11} \frac{k}{2}\left(u_{11}+u_{22}\right)_{x} .
\end{aligned}
$$


Если вычесть из (1-II) (9-II), то группа элементов $\alpha_{11} k\left(u_{22}+u_{11}\right)_{x}$ выражается только через функцию $u_{13}$,

$$
\alpha_{11} k\left(u_{11}+u_{22}\right)_{x}=\left(\frac{\alpha_{32} \alpha_{21}}{4 \alpha_{11}^{2}} \ln u_{13}-\frac{\alpha_{31}}{\alpha_{11}}-\frac{\alpha_{21} \alpha_{32}}{4 \alpha_{11}^{2}}\right) u_{13 z}-\frac{1}{k}\left(\ln u_{13}\right)_{z z} .
$$

Выполним подстановку найденного выражения, тогда в (1III), (5-III), (9-III) выделились полные производные по переменной $z$. Тем самым функции $u_{11}, u_{22}$ однозначно определились через $u_{13}$

$$
\begin{aligned}
& u_{11}=\frac{\alpha_{32} \alpha_{21}}{8 \alpha_{11}^{2}} u_{13} \ln u_{13}+\frac{1}{2 k}\left(\ln u_{13}\right)_{z}-\frac{1}{2} \alpha_{11}\left(\ln u_{13}\right)_{x}, \\
& u_{22}=-\frac{1}{2}\left(\frac{\alpha_{31}}{\alpha_{11}}+\frac{\alpha_{21} \alpha_{32}}{4 \alpha_{11}^{2}}\right) u_{13}-\frac{\alpha_{32} \alpha_{21}}{8 \alpha_{11}^{2}}\left(1+\ln u_{13}\right) u_{13}-\frac{1}{2 k}\left(\ln u_{13}\right)_{z}-\frac{1}{2} \alpha_{11}\left(\ln u_{13}\right)_{x},
\end{aligned}
$$

В результате осталось только три нетривиальных равенства

$$
\begin{aligned}
& u_{21 z}=\frac{\alpha_{21}}{2 k \alpha_{11}}\left(\ln u_{13}\right)_{z z}-u_{21}\left(\ln u_{13}\right)_{z}+\frac{\alpha_{21}}{2 \alpha_{11}}\left(\frac{\alpha_{31}}{\alpha_{11}}+\frac{\alpha_{21} \alpha_{32}}{2 \alpha_{11}^{2}}\right) u_{13 z}+\frac{\alpha_{21}}{2 k \alpha_{11}}\left(\ln u_{13}\right)_{z}^{2}, \\
& u_{31 z}=-\left(\ln u_{13}\right)_{z}\left(u_{32} \frac{\alpha_{21}}{2 \alpha_{11}}-u_{21} \frac{\alpha_{32}}{2 \alpha_{11}}-\frac{\alpha_{31}}{2 \alpha_{11} k}\left(\ln u_{13}\right)_{z}+u_{31}\right)-\frac{\alpha_{32} \alpha_{21}}{2 \alpha_{11}}\left(\ln u_{13}\right)_{z x}- \\
& -\frac{\alpha_{31}}{2 \alpha_{11}}\left(\frac{\alpha_{32} \alpha_{21}}{4 \alpha_{11}^{2}} \ln u_{13}-\frac{\alpha_{31}}{\alpha_{11}}-\frac{\alpha_{21} \alpha_{32}}{4 \alpha_{11}^{2}}\right) u_{13 z}+\frac{\alpha_{31}}{2 \alpha_{11} k}\left(\ln u_{13}\right)_{z z}, \\
& u_{32 z}=\frac{\alpha_{32}}{2 k \alpha_{11}}\left(\ln u_{13}\right)_{z z}+\alpha_{32}\left(\ln u_{13}\right)_{z x}+\frac{\alpha_{32}}{2 \alpha_{11}}\left(\frac{\alpha_{31}}{2 \alpha_{11}}+\frac{\alpha_{21} \alpha_{32}}{4 \alpha_{11}^{2}}\right) u_{13 z}- \\
& -\frac{\alpha_{32}}{4 \alpha_{11}}\left(\frac{\alpha_{31}}{\alpha_{11}}+\frac{\alpha_{32} \alpha_{21}}{\alpha_{11}^{2}}\right) u_{13 z} \ln u_{13} .
\end{aligned}
$$

Равенство (4-IV) является линейным относительно $u_{21}$, его решение имеет вид

$$
u_{21}=\frac{\alpha_{21}}{2 k \alpha_{11}} u_{13 z}+\frac{\alpha_{21}}{4 \alpha_{11}}\left(\frac{\alpha_{31}}{\alpha_{11}}+\frac{\alpha_{21} \alpha_{32}}{2 \alpha_{11}^{2}}\right) u_{13} .
$$

Интегрирование (8-IV) определяет функцию $u_{32}$ :

$$
\begin{aligned}
& u_{32}=\frac{\alpha_{32}}{2 \alpha_{11}}\left(\frac{\alpha_{31}}{\alpha_{11}}+\frac{3 \alpha_{21} \alpha_{32}}{4 \alpha_{11}^{2}}\right) u_{13}-\frac{\alpha_{32}}{4 \alpha_{11}}\left(\frac{\alpha_{31}}{\alpha_{11}}+\frac{\alpha_{32} \alpha_{21}}{\alpha_{11}^{2}}\right) u_{13} \ln u_{13}+ \\
& +\frac{\alpha_{32}}{2 k \alpha_{11}}\left(\ln u_{13}\right)_{z}+\alpha_{32}\left(\ln u_{13}\right)_{x} .
\end{aligned}
$$

В результате функция $u_{31}$ осталась произвольной, поэтому доопределим ее так

$$
u_{31}=\frac{\alpha_{21} \alpha_{32}}{4 k \alpha_{11}^{2}}\left(u_{13}-\ln u_{13}\right)_{z} .
$$


Выполним подстановку в последнее оставшееся равенство (7-IV) и получим (15).

На первый взгляд уравнение (15) удовлетворяет всем требованиям, но если умножить все члены на $u_{13}$, то тогда можно выполнить интегрирование по комбинированной переменной $z$, что приводит к уравнению первого порядка.

Теорема 2: $\quad$ Операторное уравнение Лакса (1) с дифференциальными операторами первого порядка (4) с коэффициентами, удовлетворяюшими леммам 1, 2, 3, эквивалентно нелинейному уравнению с частными производными

$$
\frac{\alpha_{21}}{2 \alpha_{11}^{2}} u_{12 z}+\frac{1}{k \alpha_{11}}\left(\ln u_{12}\right)_{z z}=k \alpha_{11}\left(\ln u_{12}\right)_{x x} .
$$

Доказательство: Очевидно, что исходная система имеет вид (1-I),.., (9I) предыдущей теоремы. Для того чтобы ход построения уравнения отличался от предыдущего случая, положим

$$
u_{13}=0 \text {, }
$$

при этом $\quad(6-\mathrm{I})$ дает $u_{23 z}=0$ тогда положим $u_{23}=0$.

В системе остаются равенства

$$
\begin{aligned}
& u_{11 z}=\frac{\alpha_{21}}{2 \alpha_{11}} u_{12}\left(2 v_{11}+k\left(u_{22}-u_{11}\right)\right)+\alpha_{11} v_{11 x}-k \alpha_{11} u_{11 x}, \\
& u_{12 z}=-u_{12}\left(2 v_{11}+k\left(u_{22}-u_{11}\right)\right), \\
& u_{21 z}=\left[u_{21}+\left(u_{22}-u_{11}\right) \frac{\alpha_{21}}{2 \alpha_{11}}\right]\left(2 v_{11}+k\left(u_{22}-u_{11}\right)\right)-\frac{\alpha_{21}}{2} k\left(u_{22}+u_{11}\right)_{x}, \\
& u_{22 z}=-\frac{\alpha_{21}}{2 \alpha_{11}} u_{12}\left(2 v_{11}+k\left(u_{22}-u_{11}\right)\right)+\alpha_{11} v_{11 x}+k \alpha_{11} u_{22 x}, \\
& u_{31 z}=\left(u_{32} \frac{\alpha_{21}}{2 \alpha_{11}}-u_{21} \frac{\alpha_{32}}{2 \alpha_{11}}+\frac{\alpha_{31}}{2 \alpha_{11} k}\left[2 v_{11}+k\left(u_{22}-u_{11}\right)\right]+u_{31}\right)\left(2 v_{11}+k\left(u_{22}-u_{11}\right)\right)+ \\
& +\frac{\alpha_{32} \alpha_{21}}{2 \alpha_{11}}\left(2 v_{11}+k\left(u_{22}-u_{11}\right)\right)_{x}-\frac{\alpha_{31}}{2} k\left(u_{22}+u_{11}\right)_{x}, \\
& u_{32 z}=\frac{\alpha_{31}}{2 \alpha_{11}} u_{12 z}-\frac{\alpha_{32}}{k \alpha_{11}}\left(\ln u_{12}\right)_{z} v_{11}-\alpha_{32}\left(2 v_{11}+k\left[u_{22}-u_{11}\right]\right)_{x}-\frac{\alpha_{32}}{2} k\left(u_{22}+u_{11}\right)_{x}, \\
& u_{22 z}+\frac{2}{k} v_{11 z}=\alpha_{11} v_{11 x}+\alpha_{11} k u_{22 x} .
\end{aligned}
$$

Функцию $u_{12}(x, z)$ будем считать отличной от нуля вместе с ее производными и постараемся выразить остальные неизвестные функции через нее. 
Разделим третье уравнение (2-II) системы на $u_{12}$, откуда находим

$$
-\left(\ln u_{12}\right)_{z}=2 v_{11}+k\left(u_{22}-u_{11}\right)
$$

Приравнивая (5-II) и (9-II), получаем

$$
v_{11}=-\frac{\alpha_{21} k}{4 \alpha_{11}} u_{12}
$$

равенство (29) определяет разность функций $u_{22}-u_{11}$

$$
u_{22}-u_{11}=\frac{\alpha_{21}}{2 \alpha_{11}} u_{12}-\frac{1}{k}\left(\ln u_{12}\right)_{z} .
$$

С учетом найденных значений система перепишется в виде

$u_{11 z}=-\frac{\alpha_{21}}{2 \alpha_{11}} u_{12 z}-\frac{\alpha_{21} k}{4} u_{12 x}-k \alpha_{11} u_{11 x}$

$u_{21 z}=-\left(\frac{\alpha_{21}}{2 \alpha_{11}}\right)^{2} u_{12 z}-\left(\ln u_{12}\right)_{z}\left[u_{21}-\frac{\alpha_{21}}{2 k \alpha_{11}}\left(\ln u_{12}\right)_{z}\right]-\frac{\alpha_{21}}{2} k\left(u_{22}+u_{11}\right)_{x}$,

$u_{22 z}=\frac{\alpha_{21}}{2 \alpha_{11}} u_{12 z}-\frac{\alpha_{21} k}{4} u_{12 x}+k \alpha_{11} u_{22 x}$,

$u_{31 z}=-\left(\ln u_{12}\right)_{z}\left(u_{32} \frac{\alpha_{21}}{2 \alpha_{11}}-u_{21} \frac{\alpha_{32}}{2 \alpha_{11}}-\frac{\alpha_{31}}{2 \alpha_{11} k}\left(\ln u_{12}\right)_{z}+u_{31}\right)-$

$-\frac{\alpha_{32} \alpha_{21}}{2 \alpha_{11}}\left(\ln u_{12}\right)_{z x}-\frac{\alpha_{31}}{2} k\left(u_{22}+u_{11}\right)_{x}$,

$u_{32 z}=\left(\frac{\alpha_{31}}{2 \alpha_{11}}+\frac{\alpha_{21} \alpha_{32}}{4 \alpha_{11}^{2}}\right) u_{12 z}+\alpha_{32}\left(\ln u_{12}\right)_{z x}-\frac{\alpha_{32}}{2} k\left(u_{22}+u_{11}\right)_{x}$,

Разность (5-III) и (1-III) позволяет найти сумму производных $u_{22}, u_{11}$

$$
u_{22 x}+u_{11 x}=-\frac{1}{k^{2} \alpha_{11}}\left(\ln u_{12}\right)_{z z}-\frac{\alpha_{21}}{2 k \alpha_{11}^{2}} u_{12 z}
$$

при подстановки в (4-III), остается связь между $u_{21}, u_{12}$ которая дает

$$
u_{21}=\frac{\alpha_{21}}{2 k \alpha_{11}} \frac{u_{12 z}}{u_{12}} \text {. }
$$

Равенство (8-III), после использования (32), позволяет найти функцию $u_{12}$

$$
u_{32}=\left(\frac{\alpha_{31}}{2 \alpha_{11}}+\frac{\alpha_{21} \alpha_{32}}{2 \alpha_{11}^{2}}\right) u_{12}+\alpha_{32}\left(\ln u_{12}\right)_{x}+\frac{\alpha_{32}}{2} \frac{1}{k \alpha_{11}}\left(\ln u_{12}\right)_{z}
$$

В результате порядок системы снижен до трех 


$$
\begin{aligned}
& u_{11 z}=-\frac{\alpha_{21}}{2 \alpha_{11}} u_{12 z}-\frac{\alpha_{21} k}{4} u_{12 x}-k \alpha_{11} u_{11 x}, \\
& u_{22 z}=\frac{\alpha_{21}}{2 \alpha_{11}} u_{12 z}-\frac{\alpha_{21} k}{4} u_{12 x}+k \alpha_{11} u_{22 x}, \\
& \left(u_{12} u_{31}\right)_{z}=-\frac{\alpha_{32} \alpha_{21}}{2 \alpha_{11}} u_{12 z x}+\frac{\alpha_{31}}{2 k \alpha_{11}} u_{12 z z}-\frac{\alpha_{21}}{2 \alpha_{11}} \frac{\alpha_{21} \alpha_{32}}{2 \alpha_{11}^{2}} u_{12} u_{12 z},
\end{aligned}
$$

причем последнее равенство интегрируется и дает возможность определить $u_{31}$

$u_{31}=\frac{\alpha_{31}}{2 k \alpha_{11}} \frac{u_{12 z}}{u_{12}}-\frac{\alpha_{32} \alpha_{21}}{2 \alpha_{11}} \frac{u_{12 x}}{u_{12}}-\frac{\alpha_{21}^{2} \alpha_{32}}{8 \alpha_{11}^{3}} u_{12}$

Последняя пара (1-IV), (5-IV) после перекрестного дифференцирования и избавиться от функции $u_{11}$, дает одно равенство на функцию $u_{12}$

$$
\frac{\alpha_{21}}{2 \alpha_{11}^{2}} u_{12 z z}+\frac{1}{k \alpha_{11}}\left(\ln u_{12}\right)_{z z z}=k \alpha_{11}\left(\ln u_{12}\right)_{z x x}
$$

которое можно понизить на порядок и получить (27).

\section{Результаты исследований и их обсуждение} Обсудим некоторые следствия, являющиеся результатом доказанных теорем

Следствие. Уравнение в частных производных (27) обладает парой Лакса (4) с матричными коэффициентами $3 \times 3, \alpha_{21}, \alpha_{11}, k-$ произвольные параметры $\alpha_{31}=\alpha_{32}$, $=1$, остальные элементы матричных коэффициентов имеют вид (11), (12), функииональные члены выражаются через $u_{12}(x, t)$ и имеют вид:

$$
\begin{aligned}
& u_{11}=\frac{1}{2 k}\left(\ln u_{12}\right)_{z}-\frac{\alpha_{21}}{4 \alpha_{11}} u_{12}-\frac{1}{2} \alpha_{11}\left(\ln u_{12}\right)_{x}, u_{13}=0, u_{21}=\frac{\alpha_{21}}{2 k \alpha_{11}} \frac{u_{12 z}}{u_{12}}, u_{23}=0, \\
& u_{31}=\frac{1}{2 k \alpha_{11}} \frac{u_{12 z}}{u_{12}}-\frac{\alpha_{21}}{2 \alpha_{11}} \frac{u_{12 x}}{u_{12}}-\frac{\alpha_{21}^{2}}{8 \alpha_{11}^{3}} u_{12}, u_{22}=-\frac{1}{2} \alpha_{11}\left(\ln u_{12}\right)_{x}+\frac{\alpha_{21}}{4 \alpha_{11}} u_{12}-\frac{1}{2 k}\left(\ln u_{12}\right)_{z}, \\
& u_{32}=\left(\frac{1}{2 \alpha_{11}}+\frac{\alpha_{21}}{2 \alpha_{11}^{2}}\right) u_{12}+\left(\ln u_{12}\right)_{x}+\frac{1}{2 k \alpha_{11}}\left(\ln u_{12}\right)_{z}, v_{11}=-v_{22}=v_{33}=-\frac{\alpha_{21} k}{4 \alpha_{11}} u_{12}, \\
& u_{33}=-\frac{1}{2} \alpha_{11}\left(\ln u_{12}\right)_{x}-\frac{1}{2 k}\left(\ln u_{12}\right)_{z}-\frac{\alpha_{21}}{4 \alpha_{11}} u_{12}, v_{23}=v_{13}=v_{21}=0, v_{12}=k u_{12}, \\
& v_{31}=-\frac{\alpha_{21}}{2 \alpha_{11}} k \frac{u_{12 x}}{u_{12}}-\frac{\alpha_{21}^{2} k}{8 \alpha_{11}^{3}} u_{12}, \quad v_{32}=\left(\frac{1}{2 \alpha_{11}}+\frac{\alpha_{21}}{2 \alpha_{11}^{2}}\right) k u_{12}+k\left(\ln u_{12}\right)_{x},
\end{aligned}
$$


для компактности записи производных введен дифференцчиальный оператор (16).

Теорема 3. Нелинейное уравнение в частных производных

$$
u^{2}\left(\beta_{22} \frac{\partial}{\partial x}+\frac{\partial}{\partial t}\right)\left[(\ln u)_{x}-u\right]+u_{x}=0
$$

эквивалентно операторному уравнению Лакса (1) с дифференцииальньми операторами вида

$$
\begin{aligned}
& L=\left(\begin{array}{ccc}
1 & 0 & 0 \\
0 & -1 & 0 \\
0 & 0 & -1
\end{array}\right) \frac{\partial}{\partial x}+\left(\begin{array}{lll}
u_{11} & u_{12} & u_{13} \\
u_{21} & u_{22} & u_{23} \\
u_{31} & u_{32} & u_{33}
\end{array}\right), \\
& A=\left(\begin{array}{ccc}
\beta_{11} & 0 & 0 \\
0 & \beta_{22} & 0 \\
0 & 0 & \beta_{22}
\end{array}\right) \frac{\partial}{\partial x}+\frac{1}{2}\left(\begin{array}{ccc}
2 v_{11} & \left(\beta_{11}-\beta_{22}\right) u_{12} & \left(\beta_{11}-\beta_{22}\right) u_{13} \\
\left(\beta_{11}-\beta_{22}\right) u_{21} & 2 v_{22} & 2 v_{23} \\
\left(\beta_{11}-\beta_{22}\right) u_{31} & 2 v_{32} & 2 v_{33}
\end{array}\right)
\end{aligned}
$$

$2 \partial e$ $u_{i j}(x, t), v_{i j}(x, t), i j=1,2,3$ - некоторые неизвестные функиии дважды дифференцируемые по своим переменныл.

Доказательство. Коэффициенты операторов (37), (38) выбраны так, что леммы 1,2 выполняются, поэтому уравнение Лакса (1) эквивалентно системе (17) выпишем ее в явном виде

$$
u_{11 t}+\beta_{11} u_{11 x}=v_{11 x}
$$

$$
\begin{aligned}
& u_{12 t}=\frac{1}{2}\left(\beta_{11}-\beta_{22}\right) u_{12}\left[u_{11}-u_{22}\right]+u_{12}\left[v_{22}-v_{11}\right]+u_{13}\left[v_{32}-\frac{1}{2}\left(\beta_{11}-\beta_{22}\right) u_{32}\right]-\frac{1}{2}\left(\beta_{11}+\beta_{22}\right) u_{12 x}, \\
& u_{13 t}=\frac{1}{2}\left(\beta_{11}-\beta_{22}\right) u_{13}\left[u_{11}-u_{33}\right]+u_{12}\left[v_{23}-\frac{1}{2}\left(\beta_{11}-\beta_{22}\right) u_{23}\right]+u_{13}\left[v_{33}-v_{11}\right]-\frac{1}{2}\left(\beta_{11}+\beta_{22}\right) u_{13 x}, \\
& u_{21 t}=u_{21}\left[v_{11}-v_{22}\right]+\frac{1}{2}\left(\beta_{11}-\beta_{22}\right) u_{21}\left[u_{22}-u_{11}\right]+\left[\frac{1}{2}\left(\beta_{11}-\beta_{22}\right) u_{23}-v_{23}\right] u_{31}-\frac{1}{2}\left(\beta_{11}+\beta_{22}\right) u_{21 x}, \\
& u_{22 t}=u_{23} v_{32}-v_{23} u_{32}-v_{22 x}-\beta_{22} u_{22 x}, \\
& u_{31 t}=u_{31}\left[v_{11}-v_{33}\right]+\left[\frac{1}{2}\left(\beta_{11}-\beta_{22}\right) u_{32}-v_{32}\right] u_{21}+\frac{1}{2}\left(\beta_{11}-\beta_{22}\right) u_{31}\left[u_{33}-u_{11}\right]-\frac{1}{2}\left(\beta_{11}+\beta_{22}\right) u_{31 x}, \\
& u_{32 t}=u_{32}\left[v_{22}-v_{33}\right]+v_{32}\left[u_{33}-u_{22}\right]-v_{32 x}-\beta_{22} u_{32 x}, \\
& u_{33 t}=u_{32} v_{23}-v_{32} u_{23}-v_{33 x}-\beta_{22} u_{33 x} .
\end{aligned}
$$


Так как число переменных превышает число уравнений, то можно ввести дополнительные связи. Пусть выполняется

$$
\begin{gathered}
v_{32}=\frac{1}{2}\left(\beta_{11}-\beta_{22}\right) u_{32}, \quad v_{23}=\frac{1}{2}\left(\beta_{11}-\beta_{22}\right) u_{23} . \\
u_{11 t}+\beta_{11} u_{11 x}=v_{11 x}, \\
\left(\frac{\beta_{11}+\beta_{22}}{2} \frac{\partial}{\partial x}+\frac{\partial}{\partial t}\right) u_{12}=\left(\frac{\beta_{11}-\beta_{22}}{2}\left(u_{11}-u_{22}\right)+v_{22}-v_{11}\right) u_{12}, \\
\left(\frac{\beta_{11}+\beta_{22}}{2} \frac{\partial}{\partial x}+\frac{\partial}{\partial t}\right) u_{13}=\left(\frac{\beta_{11}-\beta_{22}}{2}\left(u_{11}-u_{33}\right)+v_{33}-v_{11}\right) u_{13}, \\
\left(\frac{\beta_{11}+\beta_{22}}{2} \frac{\partial}{\partial x}+\frac{\partial}{\partial t}\right) u_{21}=\left(\frac{\beta_{11}-\beta_{22}}{2}\left(u_{22}-u_{11}\right)+v_{11}-v_{22}\right) u_{21}, \\
\left(\frac{\beta_{11}+\beta_{22}}{2} \frac{\partial}{\partial x}+\frac{\partial}{\partial t}\right) u_{23}=\left(\frac{\beta_{11}-\beta_{22}}{2}\left(u_{22}-u_{33}\right)+v_{33}-v_{22}\right) u_{23}, \\
\left(\frac{\beta_{11}+\beta_{22}}{2} \frac{\partial}{\partial x}+\frac{\partial}{\partial t}\right) u_{31}=\left(\frac{\beta_{11}-\beta_{22}}{2}\left(u_{33}-u_{11}\right)+v_{11}-v_{33}\right) u_{31}, \\
\left(\frac{\beta_{11}+\beta_{22}}{2} \frac{\partial}{\partial x}+\frac{\partial}{\partial t}\right) u_{32}=\left(\frac{\beta_{11}-\beta_{22}}{2}\left(u_{33}-u_{22}\right)+v_{22}-v_{33}\right) u_{32}, \\
u_{33 t}=-v_{33 x}-\beta_{22} u_{33 x} \cdot
\end{gathered}
$$

По условию теоремы $u_{12}, u_{13}, u_{21}, u_{31}, u_{32}$ отличны от нуля, тогда можем выполнить деление уравнений $(2,3,4,6,7,8$-II) на эти функции соответственно:

$$
\begin{aligned}
& \frac{\beta_{11}-\beta_{22}}{2}\left(u_{11}-u_{22}\right)+v_{22}-v_{11}=\left(\frac{\beta_{11}+\beta_{22}}{2} \frac{\partial}{\partial x}+\frac{\partial}{\partial t}\right) \ln u_{12}, \\
& \frac{\beta_{11}-\beta_{22}}{2}\left(u_{11}-u_{33}\right)+v_{33}-v_{11}=\left(\frac{\beta_{11}+\beta_{22}}{2} \frac{\partial}{\partial x}+\frac{\partial}{\partial t}\right) \ln u_{13}, \\
& \frac{\beta_{11}-\beta_{22}}{2}\left(u_{22}-u_{11}\right)+v_{11}-v_{22}=\left(\frac{\beta_{11}+\beta_{22}}{2} \frac{\partial}{\partial x}+\frac{\partial}{\partial t}\right) \ln u_{21}, \\
& \frac{\beta_{11}-\beta_{22}}{2}\left(u_{22}-u_{33}\right)+v_{33}-v_{22}=\left(\frac{\beta_{11}+\beta_{22}}{2} \frac{\partial}{\partial x}+\frac{\partial}{\partial t}\right) \ln u_{23},
\end{aligned}
$$




$$
\begin{aligned}
& \frac{\beta_{11}-\beta_{22}}{2}\left(u_{33}-u_{11}\right)+v_{11}-v_{33}=\left(\frac{\beta_{11}+\beta_{22}}{2} \frac{\partial}{\partial x}+\frac{\partial}{\partial t}\right) \ln u_{31}, \\
& \frac{\beta_{11}-\beta_{22}}{2}\left(u_{33}-u_{22}\right)+v_{22}-v_{33}=\left(\frac{\beta_{11}+\beta_{22}}{2} \frac{\partial}{\partial x}+\frac{\partial}{\partial t}\right) \ln u_{32},
\end{aligned}
$$

Попарные суммы (2-III) и (4-III), (3-III) и (7-III), (6-III) и (8-III) приводят к обратно пропорциональной связи функций

$$
u_{12}=\frac{1}{u_{21}}, \quad u_{13}=\frac{1}{u_{31}}, \quad u_{32}=\frac{1}{u_{23}}
$$

Найдем сумму (1-II), (5-II) и приведем ее к виду

$$
\left(\frac{\beta_{11}+\beta_{22}}{2} \frac{\partial}{\partial x}+\frac{\partial}{\partial t}\right)\left(u_{11}+u_{22}\right)=\left(\frac{\beta_{11}-\beta_{22}}{2}\left(u_{22}-u_{11}\right)+v_{11}-v_{22}\right)_{x} .
$$

Выполним замену правой части на выражение (4-III)

$$
\begin{gathered}
\left(\frac{\beta_{11}+\beta_{22}}{2} \frac{\partial}{\partial x}+\frac{\partial}{\partial t}\right)\left(u_{11}+u_{22}\right)=\left(\frac{\beta_{11}+\beta_{22}}{2} \frac{\partial}{\partial x}+\frac{\partial}{\partial t}\right)\left(\ln u_{21}\right)_{x}, \\
\text { следовательно, можно определить } \\
u_{11}=\left(\ln u_{21}\right)_{x}-u_{22} .
\end{gathered}
$$

Аналогично разность (5-II), (9-II) дает

$$
\left(\frac{\beta_{11}+\beta_{22}}{2} \frac{\partial}{\partial x}+\frac{\partial}{\partial t}\right)\left(u_{22}-u_{33}\right)=\left(v_{33}-v_{22}+\frac{\beta_{11}-\beta_{22}}{2}\left(u_{22}-u_{33}\right)\right)_{x} .
$$

Замена правой части на выражение (6-III) приводит к виду

$$
\left(\frac{\beta_{11}+\beta_{22}}{2} \frac{\partial}{\partial x}+\frac{\partial}{\partial t}\right)\left(u_{22}-u_{33}\right)=\left(\frac{\beta_{11}+\beta_{22}}{2} \frac{\partial}{\partial x}+\frac{\partial}{\partial t}\right)\left(\ln u_{23}\right)_{x},
$$

поэтому можно положить

$$
u_{33}=u_{22}-\left(\ln u_{23}\right)_{x} .
$$

Выполним замену найденных функций в системе

$$
\begin{gathered}
\left(\ln u_{12}\right)_{x t}+u_{22 t}+\beta_{11}\left[\left(\ln u_{12}\right)_{x}+u_{22}\right]_{x}+v_{11 x}=0, \\
v_{11}-v_{22}+\left(\beta_{11} \frac{\partial}{\partial x}+\frac{\partial}{\partial t}\right) \ln u_{12}+\left(\beta_{11}-\beta_{22}\right) u_{22}=0, \\
\left(\frac{\beta_{11}+\beta_{22}}{2} \frac{\partial}{\partial x}+\frac{\partial}{\partial t}\right) \ln u_{13}=\frac{\beta_{11}-\beta_{22}}{2}\left[\left(\ln u_{23}\right)_{x}-\left(\ln u_{12}\right)_{x}-2 u_{22}\right]+v_{33}-v_{11}, \\
u_{22 t}=-v_{22 x}-\beta_{22} u_{22 x},
\end{gathered}
$$




$$
\begin{array}{r}
v_{22}-v_{33}+\left(\beta_{22} \frac{\partial}{\partial x}+\frac{\partial}{\partial t}\right) \ln u_{23}=0, \\
u_{22 t}-\left(\ln u_{23}\right)_{x t}=-v_{33 x}-\beta_{22}\left[u_{22}-\left(\ln u_{23}\right)_{x}\right]_{x}
\end{array}
$$

Если из (6-IV) выразить $v_{22}$, а из (2-IV) функцию $v_{11}$ и подставить в (3-IV), то можно вынести дифференциальный оператор за скобки

$$
\left(\frac{\beta_{11}+\beta_{22}}{2} \frac{\partial}{\partial x}+\frac{\partial}{\partial t}\right)\left(\ln u_{13}-\ln u_{12}-\ln u_{23}\right)=0
$$

для тождественного выполнения равенства определим следующую связь между функциями

$$
v_{13}=v_{12} v_{23} \text {. }
$$

Использование (43) приводит систему к виду

$$
\begin{gathered}
\left(\beta_{11} \frac{\partial}{\partial x}+\frac{\partial}{\partial t}\right)\left[\left(\ln u_{12}\right)_{x}+u_{22}\right]+v_{11 x}=0, \\
v_{11}-v_{22}+\left(\beta_{11} \frac{\partial}{\partial x}+\frac{\partial}{\partial t}\right) \ln u_{12}+\left(\beta_{11}-\beta_{22}\right) u_{22}=0, \\
v_{33}-v_{11}-\left(\beta_{11}-\beta_{22}\right) u_{22}-\left(\beta_{22} \frac{\partial}{\partial x}+\frac{\partial}{\partial t}\right) \ln u_{23}-\left(\beta_{11} \frac{\partial}{\partial x}+\frac{\partial}{\partial t}\right) \ln u_{12}=0, \\
u_{22 t}=-v_{22 x}-\beta_{22} u_{22 x}, \\
v_{22}-v_{33}+\left(\beta_{22} \frac{\partial}{\partial x}+\frac{\partial}{\partial t}\right) \ln u_{23}=0, \\
u_{22 t}-\left(\ln u_{23}\right)_{x t}=-v_{33 x}-\beta_{22}\left[u_{22}-\left(\ln u_{23}\right)_{x}\right]_{x} .
\end{gathered}
$$

Из (3-IV) определим

$$
v_{11}=v_{33}-\left(\beta_{22} \frac{\partial}{\partial x}+\frac{\partial}{\partial t}\right) \ln u_{23}-\left(\beta_{11} \frac{\partial}{\partial x}+\frac{\partial}{\partial t}\right) \ln u_{12}-\left(\beta_{11}-\beta_{22}\right) u_{22}
$$

В результате остались уравнения, которые после подстановки (44) совпали

$$
v_{33 x}-\left(\beta_{22} \frac{\partial}{\partial x}+\frac{\partial}{\partial t}\right)\left[\left(\ln u_{23}\right)_{x}-u_{22}\right]=0 .
$$

Положим $\quad v_{33}=\frac{1}{u_{12}}, u_{23}=u_{22}=u_{12}=u(x, t)$.

Следствие. Нелинейное уравнение в частных производных, заданное на функиию двух переменных $и(x, t)$

$$
u^{2}\left(\beta_{22} \frac{\partial}{\partial x}+\frac{\partial}{\partial t}\right)\left[(\ln u)_{x}-u\right]+u_{x}=0
$$


эквивалентно операторному уравнению Лакса (1) с дифференциальными операторами вида

$$
\begin{aligned}
& L=\left(\begin{array}{ccc}
1 & 0 & 0 \\
0 & -1 & 0 \\
0 & 0 & -1
\end{array}\right) \frac{\partial}{\partial x}+\left(\begin{array}{ccc}
-(\ln u)_{x}-u & u & u^{2} \\
u^{-1} & u & u \\
u^{-2} & u^{-1} & u-(\ln u)_{x}
\end{array}\right), \\
& A=\left(\begin{array}{ccc}
\beta_{11} & 0 & 0 \\
0 & \beta_{22} & 0 \\
0 & 0 & \beta_{22}
\end{array}\right) \frac{\partial}{\partial x}+\frac{1}{2}\left(\begin{array}{ccc}
2 v_{11} & \left(\beta_{11}-\beta_{22}\right) u & \left(\beta_{11}-\beta_{22}\right) u^{2} \\
\left(\beta_{11}-\beta_{22}\right) u^{-1} & 2 u^{-1}-\left(\beta_{22} \frac{\partial}{\partial x}+\frac{\partial}{\partial t}\right) \ln u\left(\beta_{11}-\beta_{22}\right) u \\
\left(\beta_{11}-\beta_{22}\right) u^{-2} & \left(\beta_{11}-\beta_{22}\right) u^{-1} & 2 u^{-1}
\end{array}\right), \\
& \text { где } \\
& v_{11}=u^{-1}-\left(\left[\beta_{22}+\beta_{11}\right] \frac{\partial}{\partial x}+2 \frac{\partial}{\partial t}\right) \ln u-\left(\beta_{11}-\beta_{22}\right) u
\end{aligned}
$$

\section{Выводы}

1. В статье использовано операторное уравнение Лакса для построения нелинейного уравнения в частных производных. В качестве пары Лакса выбраны дифференциальные операторы первого порядка с матричными коэффициентами $3 \times 3$.

2. Определены необходимые и достаточные условия, накладываемые на параметры и функции, при которых коммутатор двух дифференциальных операторов представляет оператор умножения.

3. Доказано, что если в операторах дифференциальный матричный коэффициент имеет нижнетреугольной вид, то система уравнений однозначно сводится к одному уравнению.

4. В случае, когда в операторах дифференциальный матричный коэффициент имеет диагональный вид, то система дифференциальных уравнений допускает вариацию функциональных переменных.

\section{Библиографический список}

1. Журавлев В.М. // ЖЭТФ, т. 110. № 6, с. 910-929. 1996.

2. Журавлев В.М. // Письма в ЖЭТФ. 61, в. 4. 254. 1995.

3. Журавлев В.М. Нелинейные волны в многокомпонентных системах с дисперсией и диффрузией. Точно решаемые модели // Ульяновск: Изд-во УлГУ, 2001. 256 с.

4. Захаров В.Е. Солитоны // под ред. Р. Буллафа, Ф. Кодри. М.: Мир, 1970. 1983.

5. Захаров В.Е., Манаков С.В., Новиков С.П., Питаевский Л.П. / под ред. С.П. Новикова. Теория солитонов: Метод обратной задачи. М.: Наука, 1980. 319 с. 
6. Карюк А.И., Редькина Т.В. Квазилинейное волновое уравнение, обладающее парой Лакса // Обозрение прикладной и промышленной математики. Т. 14, вып. 4. Москва, 2007. С. 717-718.

7. Лакс П.Д. и Ральф Ф.С. Теория рассеяния. М.: Мир, 1971. 312 с.

\section{References}

1. ZHuravlev V.M. // ZHEHTF, t. 110. № 6 s. 910-929. 1996. (in Russ)

2. ZHuravlev V.M. // Pis'ma v ZHEHTF. 61 v. 4. 254. 1995. (in Russ)

3. ZHuravlev V.M. Nelinejnye volny $v$ mnogokomponentnyh sistemah $s$ dispersiej i diffuziej. Tochno reshaemye modeli (Nonlinear waves in multicomponent systems with dispersion and diffusion. Exactly solvable models)//UI'yanovsk, Izd-vo UIGU, 2001 - 256 s. (in Russ)

4. Zaharov V.E. Solitony (Solitons) // Pod red. R.Bullafa, F.Kodri. M.Mir 270. 1983. (in Russ)

5. Zaharov V.E., Manakov S.V., Novikov S.P., Pitaevskij L.P.; Pod red. S.P. Novikova. Teoriya solitonov: Metod obratnoj zadachi (Soliton theory: The inverse problem method) // - M.: Nauka, 1980. - $319 \mathrm{~s}$. (in Russ)

6. Karyuk A.I., Red'kina T.V. Kvazilinejnoe volnovoe uravnenie, obladayushchee paroj Laksa (A quasilinear wave equation possessing a Lax pair) //Obozrenie prikladnoj i promyshlennoj matematiki. T. 14, vyp. 4. Moskva. 2007. S. 717-718 (in Russ).

7. Laks P.D. i Ral'f F.S. Teoriya rasseyaniya (Theory of scattering). M.: Mir, 1971. 312 s. (in Russ).

Рукопись поступила в редакцию: 18.05.2018, принята к публикации: 26.05.2018

\section{Сведения об авторах}

Яновская Ольга Сергеевна, аспирант кафедры общей и теоретической физики, старший преподаватель кафедры прикладной математики и математического моделирования Северо-Кавказского федерального университета.Researcher ID: Q-7014-2018 Телефон: (8652) 33-01-45. Email: olenka_yan@mail.ru.

Сурнева Олеся Борисовна, аспирант кафедры общей и теоретической фризики, Северо-Кавказского федерального университета. Researcher ID: Q-7009-2018 Телефон: 33-01-45. Email: surnevao@mail.ru.

\section{About the authors}

Yanovskaya Olga Sergeevna, postgraduate student of the Department of General and Theoretical Physics, senior lecturer of the Department of Applied Mathematics and Mathematical Modeling of the North Caucasus Federal University. Researcher ID: Q-7014-2018 Phone: (8652) 3301-45. Email: olenka_yan@mail.ru.

Surneva Olesya Borisovna, post-graduate student of the Department of General and Theoretical Physics, North-Caucasian Federal University Researcher ID: Q-7009-2018 Phone: (8652) 33-01-45. Email: surnevao@mail.ru. 